$51 \mid 2013$

Grammaire et enseignement du français langue étrangère et seconde. Permanences et ruptures du XVle au milieu du XXe siècle (I)

\title{
Enseigner/apprendre la grammaire française par demandes et réponses
}

Maria Eugenia Fernández Fraile

\section{(2) OpenEdition \\ Journals}

\section{Édition électronique}

URL : https://journals.openedition.org/dhfles/3811

DOI : $10.4000 /$ dhfles.3811

ISSN : 2221-4038

\section{Éditeur}

Société Internationale pour l'Histoire du Français Langue Étrangère ou Seconde

Édition imprimée

Date de publication : 1 février 2013

ISSN : 0992-7654

\section{Référence électronique}

Maria Eugenia Fernández Fraile, «Enseigner/apprendre la grammaire française par demandes et réponses », Documents pour l'histoire du français langue étrangère ou seconde [En ligne], 51 | 2013, mis en ligne le 31 janvier 2017, consulté le 28 mai 2021. URL : http://journals.openedition.org/dhfles/3811 ; DOI : https://doi.org/10.4000/dhfles.3811

Ce document a été généré automatiquement le 28 mai 2021.

(c) SIHFLES 


\title{
Enseigner/apprendre la grammaire française par demandes et réponses
}

\author{
Maria Eugenia Fernández Fraile
}

1 La description et la présentation de la grammaire française par demandes et réponses (ou grammaire dialoguée) constituent une démarche qui jouit d'une forte présence au cours des $\mathrm{XVI}^{\mathrm{e}}$-XIXe siècles. Le but de notre article consiste à établir ce procédé de description/ présentation textuelle dans son contexte historique, en retraçant sa filiation; à proposer un répertoire (forcément incomplet) des ouvrages de ce type (dans les limites temporelles indiquées); à analyser les particularités de ce mode de description/présentation du contenu grammatical; finalement, à insérer ce type d'ouvrages dans l'histoire de l'enseignement du français, en nous arrêtant de façon particulière sur les Principes de Restaut (1730).

\section{Filiation et typologie}

2 Constituer la filiation (ou la genèse) des grammaires par demandes et réponses nous mène obligatoirement à un classement des "dialogues ", ce qui permettra de mieux comprendre les caractéristiques de celles-ci. Le dialogue est un échange verbal entre deux ou plusieurs personnes, un type discursif opposé au monologue. Les différentes prises de parole (ou répliques) constituant alors une conversation peuvent être regroupées selon des finalités diverses. Ainsi, le dialogue peut devenir un procédé didactique, et le rapport entre maître et élève est réglé sur le mode de l'entretien oral en tête-à tête: l'enseignement à travers l'interaction dialoguée professeur-disciple remonte, comme nous le savons tous, à Socrate. Cet échange verbal se transforme, par le biais de l'écriture, en type textuel (en « dialogue ») qui revêtira une série de formes au fil du temps, à l'intérieur desquelles il faut inscrire la grammaire par demandes et réponses. Le dialogue admet ainsi trois grandes modalités (Stati 1998 : 7) :

3 a) Le dialogue en tant qu'« œuvre didactique, littéraire ou philosophique, écrite sous forme de conversation entre deux ou plusieurs interlocuteurs ou groupes d'interlocuteurs, de manière à mettre en évidence la contradiction ou, le cas échéant, la 
convergence entre les opinions, les idées, les thèses que l'auteur les charge d'exposer " (CNRTL). Ce type de genre textuel existe dès l'Antiquité classique, comme l'affirme J.-Cl. Chevalier (1998: 17) : c'est le cas notamment des Dialogues de Platon; le long des XVII et XVIII ${ }^{\mathrm{e}}$ siècles, la présentation dialoguée devient un procédé littéraire courant, utilisé par Cervantès, Fontenelle, Diderot ou Voltaire...

b) Le dialogue en tant que regroupement de prises de parole entre deux ou plusieurs personnes ayant pour but un apprentissage linguistique. La reproduction écrite d'échanges verbaux simples en langage courant (sermo pedestris), en vue d'une utilisation didactique pour apprendre le latin se faisait dès l'Antiquité classique ${ }^{1}:$ c'est $^{\prime}$ le cas des Hermeneumata, écrits vers l'an 300 par un grammairien connu sous le nom de pseudo-Dosithée. Cet outil, complémentaire des vocabulaires (nominalia, disposés par ordre de matières) accompagnés de gloses, et de plus en plus couramment sous une forme bilingue, est repris au cours du Moyen Âge, évolue - les catalogues de phrases sous forme de dialogue sont classés par catégories, cf. les Vulgaria Terentii, 1480 -, et se systématise, comme le montre J.-Cl. Chevalier (1998: 20-23). L'enseignement du latin continue de les utiliser pendant tout le XVI ${ }^{\mathrm{e}}$ siècle ( $c f$. les Colloquia d'Erasme, 1523 et années suivantes ou la Linguae latinae exercitatio, de Vivès, 1540) :

À la fin du Moyen Âge, sous l'influence de l'humanisme, le recours à ce genre de dialogues se généralise en tant que pratique didactique et s'introduit dans les cours de latin même : rédigés par des maîtres allemands, flamands, espagnols, français et suisses, ces 'colloques scolaires' se proposent de ressusciter la langue de Cicéron et de l'adapter aux besoins de la communication quotidienne des étudiants des Universités [...]. (Colombo Timelli $1998: 28$ )

5 Cet outil est utilisé de même à partir du XIV ${ }^{e}$ siècle pour l'apprentissage des langues vivantes ( $c f$. le Dialogue des Mestiers, dialogues français-flamands, composé au XIV siècle, publié par H. Michelant, 1875, Paris, Lib. Tross, ou la Manière de langage qui enseigne à parler et à écrire le français, composée en 1396, publiée par P. Meyer, 1870), en l'adaptant aux destinataires présumés («juristes, commerçants, militaires [...]», Chevalier 1998 : 18), et constitue l'ouvrage-type ou représentatif $\mathrm{du} \mathrm{XVI}^{\mathrm{e}}$ siècle dans le domaine de l'enseignement des langues vivantes ( $c f$. les œuvres de G. du Wez, Noel de Berlaimont, G. Meurier...). Les dictionnaires plurilingues des $\mathrm{XVI}^{\mathrm{e}}$ et $\mathrm{XVII}{ }^{\mathrm{e}}$ siècles (Berlaimont, Solenissimo Vocabulista...) incorporent aussi fréquemment des dialogues et des phraséologies (cf. Colombo Timelli 1998 : 28). Comme l'indique J.-Cl. Chevalier, ce qu'on appelle colloques «[...] sont directement apparentés aux grammaires, si théoriques qu'elles se prétendent, aux listes de vocabulaire, quelle qu'en soit l'économie » (1998: 24).

6 Les « dialogues familiers » seront une composante habituelle des grammaires destinées à l'enseignement des langues vivantes aux XVII ${ }^{e}$ et XVIII ${ }^{e}$ siècles ; ils peuvent constituer aussi un ouvrage à part, proposé comme outil complémentaire à une grammaire et à un dictionnaire (cf. Oudin, 1608 ; Juan de Luna, 1621 ; Sobrino, 1708, etc.). N. Minerva et J. Lillo ont étudié de façon précise la présence des «dialogues» dans les grammaires et les manuels pour apprendre le français publiés en Italie (1998: 83-98 et 99-124). Même de nos jours, on utilise à des fins pédagogiques des variantes de dialogues tels que l'interview, le débat, l'entretien sur un sujet, le bavardage sur des thèmes courants, le dialogue théâtral (Pellandra 1998: 143-156). Nous n'insistons pas sur ce point, et renvoyons au numéro de Documents dirigé par N. Minerva consacré à cette question $\left(1998, n^{\circ} 22\right)$. 
7 c) Le dialogue peut être utilisé également comme moyen de présentation du contenu d'une discipline ou d'une matière scientifique, de façon simplifiée ou dans toute la gamme de sa complexité. Ce procédé date également du Moyen Âge (Chevalier 1998 : 17 ; Colombo Timelli 1998 : 27). P. Meyer - l'éditeur de la Manière de langage écrit en 1396 - se fait l'écho d'une série de traités ou d'opuscules, ayant pour objet l'enseignement du latin (et aussi du français, de façon complémentaire), et utilisant fréquemment le français comme langue véhiculaire (composés par Alexandre Neckam, J. de Garlande...), et qui sont composés « pour enseigner la valeur, le genre, l'orthographe des mots ». Et il ajoute en note en pied de page : « Il y a bien eu au XIV siècle une sorte de grammaire élémentaire par demandes et réponses, rédigée en français, mais c'est une grammaire faite pour apprendre le latin et non le français; voy. Thurot, Extraits, etc., p. 53, 168, 170, $175, \ldots$... (Meyer $1870: 381$ ). L'Ars Minor de Donat est également rédigé au Moyen Âge sous forme de questions-réponses, et des traductions françaises sont faites dès la fin du $\mathrm{XV}^{\mathrm{e}}$ siècle ( $c f$. Colombo Timelli 1995 et 1996). B. Colombat fait état également de la longue tradition d'enseignement du latin par le biais des questions-réponses (erotemata) :

Le grammairien utilise une pédagogie graduée. Conformément à une tradition ancienne, il procède par questions-réponses (erotemata), avec des questions de deux types. Les unes concernent la terminologie: appel de définition (Quid est dictio ?) et justification étymologique (Unde dicta est dictio ?). Les autres assurent l'illustration (Quaestiones de dictione): appel de classement dans une catégorie (Poeta, quae uox est ?) et justification de l'appartenance à cette catégorie (Quare? [...]). (Colombat $1988: 52)$

8 C'est ce type d'ouvrage qui constitue de façon précise l'objet de notre article: les grammaires par demandes et réponses s'inscrivent en effet dans cette tradition. Nous avons ainsi affaire à une application didactique ou scolaire du type «dialogue philosophique », qui constitue un type spécifique de mise en forme du procédé du dialogue.

\section{Répertoire}

9 La présentation dialoguée de questions grammaticales et/ou linguistiques à des fins didactiques ne concerne pas uniquement le latin et le français : nous plaçons en annexe la liste alphabétique des ouvrages que nous avons pu répertorier, où nous avons incorporé quelques ouvrages portant sur la langue espagnole ou ayant un but bilingue (comparaison entre deux langues), puisqu'ils constituent des modèles qui ont sûrement exercé une influence sur les grammaires du français. Nous nous limitons à faire quelques remarques à ce sujet :

10 -si nous examinons la distribution par siècles, nous pouvons constater que l'édition d'ouvrages de ce genre "explose" au XIX siècle, probablement en raison de la disciplinarisation du français comme matière scolaire : de cinq-six ouvrages par siècle du XVI ${ }^{e}$ au XVIII ${ }^{e}$ siècle on passe à plus de cinquante au XIX ${ }^{\mathrm{e}}$ siècle ;

11 -la présentation dialoguée n'impliquait point une simplification du contenu grammatical au XVI eiècle: le Diálogo de la lengua de Valdés (1533), le Dialogue de l'orthographe... de J. Peletier du Mans (1555), la Grammaire de Ramus (1572) sont des ouvrages adressés à des adultes, des étudiants, des amateurs ou des érudits, sans que le contenu ait été réduit (en principe) du fait de la présentation dialoguée ; 

qui portent chacun leur nom (prénom) : dans le Diálogo de la lengua, de Juan de Valdés (1533), quatre personnages, nommés dans le texte Valdés, Marcio, Coriolano, Pacheco, s'entretiennent sur des questions touchant la langue, et de façon particulière la langue castillane. Cette mise en scène textuelle reprend ainsi le modèle des Dialogues de Platon. Dans le Diálogo de la lengua, l'un des personnages, nommé précisément Valdés, possède une qualité reconnue de " connaisseur ", aux yeux de ses interlocuteurs, car il est né en Espagne et c'est un lettré ; deux autres, de nationalité italienne (Coriolano, Marcio), désirent perfectionner leur connaissance de la langue castillane ; quant au quatrième, Pacheco, d'origine espagnole, il désire mieux connaître sa langue maternelle pour pouvoir briller en société en Italie, avec l'assurance de ne point se tromper dans son dire. Le contenu de la matière est exposé sous forme d'échange ou d'entretien, même si l'un d'entre eux (Valdés) possède un rôle majeur dans cette fonction. Nous ne pouvons pas ici détailler le fonctionnement discursif de ces échanges ; signalons simplement que la composition et la progression de l'ouvrage se produisent selon le schéma-modèle suivant :

a) question d'un personnage

b) prise de parole (plus ou moins longue) de la part du spécialiste

c) manifestation de l'accord de l'un ou de plusieurs interlocuteurs

d) synthèse de ce qui a été dit

e) changement de sujet.

2. Une deuxième modalité est constituée par la mise en scène de deux interlocuteurspersonnages, nommés le « précepteur » et le « disciple ». C'est le cas de la Grammaire de Pierre de la Ramée (1572). Ici, c'est toujours l'élève qui pose les questions, et le maître, seul à savoir, expose la matière, sans aucune interruption. Cette mise en scène textuelle laisse paraître son artifice, car le prétendu dialogue est totalement déséquilibré, et il devient facilement un exposé monologué de la part du maître ; de façon corrélative, les questions du disciple sont souvent des questions rhétoriques : " pourquoi doncques?", etc. La présentation de la matière grammaticale a pour but de reprendre l'essentiel du corps doctrinal de la discipline, dans la langue-cible même, sous forme d'un « contenu rigidement structuré et hiérarchisé » (Colombo Timelli 1998 : 27).

L'échange verbal y est donc simulé : un précepteur et un disciple semblent échanger des questions et des réponses. Cette mise en scène permet toutefois un fonctionnement discursif intéressant du point de vue pédagogique : c'est l'élève qui désire savoir, et c'est de lui que part l'intérêt à savoir. Le maître satisfait un besoin préexistant, qu'il ne doit pas justifier par une longue préface comme dans les grammaires, habituellement, 
où l'auteur cherche à convaincre de l'utilité de la grammaire et de la nécessité de parler des langues.

3. La présentation sous forme de demandes et réponses peut adopter également la forme de questionnaire où le maître n'expose point, mais pose les questions aux élèves qui, par le biais des réponses, exposent le contenu grammatical. C'est le cas des grammaires par demandes et réponses, dont celles de Pierre Restaut (1730) et de Juan Tomás de Mairan (1799). Ainsi, dans ce troisième type de "grammaires par demandes et réponses", la présentation de la matière s'est dénudée de son conditionnement discursif et est devenue un simple atout pédagogique. Elle se constitue ainsi comme une "stratégie didactique » de choix dans la culture scolaire occidentale (Colombo Timelli 1998 : 27), qui embrasse progressivement toutes les matières (tout particulièrement le catéchisme). La présentation sous forme de questions et réponses présente en effet de nombreux avantages pédagogiques, autant pour le maître que pour l'élève :

a) la simplification et la décomposition de la matière: l'auteur procède à une fragmentation de la matière en "unités minimales ", pourrait-on dire, en vue de sa mémorisation (face à la lectio explicative, qui constitue un bloc de contenu plus consistant). Les énoncés doivent être simples (propositions indépendantes) et clairs. L'élève sait parfaitement ce qu'il doit apprendre. La page de titre des Principes élémentaires... de Oger (1801) indique ainsi que l'ouvrage contient: «De nouveaux moyens pour classer, simplifier, éclaircir et diminuer les difficultés de la Lecture et de l'Orthographe ». Ou encore, Mme Roulleaux, dans la présentation de sa Grammaire française à l'usage des pensionnats de demoiselles (1834), déclare :

Persuadée que le défaut de classification des matières et le manque d'un ordre croissant des difficultés, ne peuvent que ralentir les progrès des enfans, et, en les fatiguant, les dégoûter de l'étude, j'ai cru qu'un exposé clair et précis des règles et un ordre méthodique [...] devaient porter des fruits. Telle a été ma pensée avant d'écrire cette grammaire, dans laquelle j'ai gradué les épines d'une langue, dont j'ai cru faciliter l'étude par une série de demandes et réponses (1834: Introduction)

Les qualités pédagogiques des grammaires par demandes et réponses sont explicitées par Pierre Restaut de façon très claire, et leur exposé constitue également un atout pour convaincre les lecteurs. Sa préface a en effet pour but de ne point rebuter un lecteur qui n'aurait point fait d'études latines: il ne parle pas de règles, mais de principes; il va commencer son texte par les principes généraux (à travers lesquels il fournit la compréhension des notions grammaticales de base ainsi que le métalangage correspondant), en s'appuyant sur un auteur de prestige de l'époque tel que Rollin :

Il n'y a personne qui ne convienne que l'on n'avance dans quelque science que ce puisse être, qu'autant qu'on en a étudié \& approfondi les véritables principes : ce qui me donne lieu d'assurer, après l'excellent* Auteur De la manière d'enseigner \& d'étudier les Belles Lettres que la méthode la plus courte \& en même temps la plus solide d'apprendre une langue est de s'y préparer par une connaissance exacte et raisonnée de ces principes généraux \& particuliers, en les appliquant à la langue que l'on sait déjà par habitude. (Restaut 1730 : Préface, III)

Donc, la grammaire doit être simplifiée, limitée à la connaissance des principes essentiels. Ce qu'il observe par contre, dans les grammaires de l'époque, c'est que

elles sont trop chargées \& qu'elles ne sont point assez simples pour les personnes sans études \& sur tout pour les Dames, qui sont d'abord rebutées par la nouveauté des termes, \& effrayées par l'abondance de matières. [...] [Il faudrait donc] une Méthode courte $\&$ facile, où elles ne trouvassent que des principes généraux, suivis \& raisonnés. (Ibid. : XXj) 
Il compose ainsi en 1732 un Abrégé des Principes, où "tout sera simple et facile» (Restaut 1730: Préface, XV). La rédaction donc en langue-cible se couple d'une présentation textuelle des principes dans le style dialogué : « plus propre que tout autre à mettre une liaison naturelle entre les principes \& les conséquences, les objectifs \& et les réponses. » (Ibid. : X)

Ces principes, il va les soumettre à la raison, et les exposer dans l'ordre le plus simple qui soit ; s'il y a des notions difficiles, il a eu « le soin de les éclairer par les explications simples \& familières » (ibid., IX). Il élimine également beaucoup de difficultés qui sont produites par une disposition de la matière inadéquate dans les grammaires antérieures: " on y suppose, par exemple, la connaissance des Noms en parlant des articles, celle des Verbes dans le Traité des Pronoms. » (Ibid. : X)

b) la matière, présentée sous forme de questionnaire, est plus facile à mémoriser de la part de l'élève, et, de même, le contrôle de la leçon (apprise par l'élève) de la part du maître est également plus aisé : le maître n'a qu'à formuler des demandes déjà toutes faites; l'élève est guidé par la question du maître : il sait exactement ce qu'il doit répondre, s'il a appris sa leçon. Dans les catéchismes, le procédé textuel met en scène deux personnages (maître et élève) où celui-ci a déjà assimilé la matière/contenu à apprendre, puisqu'il est capable de réciter sa leçon de façon parfaite !

Le modèle pédagogique de référence est en principe aux antipodes de la méthode cartésienne, et même du dialogue philosophique: dans ces cas, on prétend faire comprendre le corps doctrinal de la matière traitée au moyen d'explications qui ont pour but de générer la compréhension de la matière ou d'en débattre (presque d'égal à égal). Dans la grammaire par demandes et réponses, en revanche, on ne cherche pas l'association de l'élève au processus de connaissance par la compréhension du contenu (fonction assignée aux explications que fournit le maître), mais uniquement sa mémorisation du contenu (même sans le comprendre) et sa répétition psittacique. On y apprend la grammaire comme un catéchisme ; la mémoire y joue le principal rôle et la réflexion trouvait peu ou ne trouvait rien sur quoi s'exercer. Ainsi, il n'existe point d'exemples qui illustrent les règles apprises. Certains auteurs de "grammaires par demandes ou réponses ", cependant, tel Oger (1801), essaieront d'y intégrer des explications, sous forme de notes en pied de page, ou de textes complémentaires qui fonctionnent comme des ajouts (remarques, etc.).

1 Aussi, dans sa présentation ou son fonctionnement textuel, la grammaire par demandes et réponses inverse-t-elle la situation d'enseignement-apprentissage ; certains ouvrages mettent en scène la situation discursive suivante, où les personnages réels (précepteur, pédagogue, maître et disciple, élève, etc.) disparaissent au profit d'une simple indication de "demande » et "réponse ", et où c'est l'élève qui donne la réponse ! Le texte anticipe le résultat prétendu, et explicite en toutes lettres ce que l'élève doit avoir appris, contenu qui correspond parfaitement avec le contenu de la matière.

Si chez Mauger \& Festeau (1693/1696), il existe encore une mise en discours qui feint un dialogue pédagogique (l'élève questionne, par exemple: «je voudrais bien sçavoir [...]», le maître répond, voir p. 44), chez Restaut, Coffigny ou Mme Roulleaux, les personnages ont disparu au profit d'une simple indication de rôle (D et R), et la présentation du contenu n'est plus faite par le maître (qui répond à une demande supposée de l'élève) mais par l'élève lui-même (qui a mémorisé la réponse), ainsi : « D. 
Qu'est-ce l'article ? - R. C'est un mot qui se met devant un nom [...]» (Restaut 1730: 8 ; Coffigny $1828: 10$ ).

\section{Conclusion} demandes et réponses se développe amplement au XIXe siècle : ce sont certainement les Principes et l'Abrégé de Restaut qui ont redonné une seconde vie à ce type d'ouvrages, en se constituant comme modèle de référence pour les ouvrages postérieurs.

Les raisons sont multiples. Aux yeux de Restaut, le grand atout de la grammaire par demandes et réponses est qu'elle permet l'apprentissage de la langue (maternelle et étrangère, sans distinction) de la part de gens non instruits en latin, ne possédant pas les notions grammaticales de base, et donc de tous «[...] ceux qui ne l'ont jamais apprise par les règles» (Restaut 1730 : Préface : I). Et c'est à cette cible qu'il adresse principalement ses ouvrages: les Principes et l'Abrégé des principes. Il dit ainsi que son ouvrage est utile aux enfants (qui vont ou ne vont pas apprendre le latin par la suite), aux étrangers, mais aussi aux Français (Préface, XII-XXIV). Bien sûr, au-delà de la composante publicitaire dont participe la Préface des Principes de Restaut, il reste que des couches/catégories de la population qui jusque-là ne faisaient point d'études linguistiques, ou qui en faisaient de façon incorrecte selon lui (ne possédant pas des outils adéquats) vont participer à l'étude de la langue française.

D'autre part, cette présentation du contenu rompt avec les pratiques de l'époque quant à l'enseignement des langues d'une façon radicale: tout d'abord, en montrant l'absurdité de ces pratiques : «A peine savent-ils [les enfants] lire, que sans leur avoir donné aucune notion de leur Langue naturelle, on les met tout d'un coup dans les principes d'une langue qui leur est absolument étrangère " (Restaut 1730 : Préface, V). Restaut prend parti pour un apprentissage grammatical/linguistique basé sur la langue maternelle (et non pas sur le latin),

la méthode la plus courte \& en même temps la plus solide d'apprendre une langue est de s'y préparer par une connaissance exacte \& raisonnée de ces principes généraux et particuliers en les appliquant à la langue qu'on fait déjà par habitude. (Restaut 1730 : Préface, III)

Le besoin d'un apprentissage de la langue maternelle à travers une grammaire est pour lui incontestable : «Il faut donc avoir recours aux Grammaires » (1730 : Préface, XXI).

Mais aussi, la grammaire par demandes et réponses permet l'apprentissage des règles d'une langue (maternelle ou étrangère) et en même temps, l'acquisition de la "méchanique" de la langue, c'est-à-dire, des combinaisons des mots (syntaxe, phraséologie), question essentielle dans la connaissance d'une langue :

Le raisonnement seul ne suffit pas pour l'étude d'une langue. Il faut encore que la mémoire se charge \& remplisse d'un grand nombre de mots \& de combinaisons différentes, dont la connoissance ne s'acquiert que par un exercice continué \& peut être du ressort d'aucune méchanique. (Restaut 1730 : Préface, VIII)

C'est un atout important aux yeux de Restaut. Les grammaires pour étrangers composées dans la langue de l'apprenant, leur permettant ainsi de "comprendre » le contenu grammatical, possèdent en effet un énorme défaut: les apprenants apprennent les règles, mais pas l'usage de la langue. La proposition de Restaut réunit, à sa façon, l'apprentissage par principes et l'apprentissage par l'usage, puisque les 
apprenants pratiquent la langue étrangère dès le début. Et il n'est pas le seul à le proposer : la réunion nécessaire de ces deux démarches est posée dans l'article Langue de l'Encyclopédie ; l'Abbé Pluche fait un pas en avant, en défendant une répartition des deux voies en phases successives de l'apprentissage : si on commence par la première voie (celle des règles) et qu'ensuite on y adjoint l'usage, on emprunte un chemin « long et plein d'obstacles»; si on commence par contre par la seconde voie (celle de l'usage) et si on y adjoint ensuite les règles, « voilà la route simple et sûre » (Pluche 1751 : 40).

Nous devons donc considérer Restaut comme l'un des grands réformateurs de l'enseignement de la langue française. A. Chervel l'affirme, également, à propos de la distinction entre orthographe d'usage et orthographe par principes que Restaut établit: «La découverte de Restaut est le point de départ d'un vaste effort collectif, poursuivi jusqu'au $\mathrm{XX}^{\mathrm{e}}$ siècle, pour mettre au point une didactique de l'orthographe totalement détachée du latin, et ne faisant appel qu'aux seules ressources du français » (Chervel $2008:$ 207).

\section{BIBLIOGRAPHIE}

CARAVOLAS, Jean Antoine (1995). « Apprendre á parler une langue étrangère à la Renaissance ». Historiographia Linguistica, XXII, 3, 275-306.

CHERVEL, André (2008). Histoire de l'enseignement du français. Paris : Retz.

CHEVALIER, Jean-Claude (1998). « Les colloques médiévaux. Origines, filiations, méthodes. Quelques pistes ». Documents pour l'Histoire du Français Langue Étrangère et/ou Seconde, 22, 17-26.

CNRTL. Centre national de ressources textuelles et lexicales.

[http://www.cnrtl.fr/definition/dialogue].

COLOMBAT, Bernard (1988). « Les 'parties du discours' (partes orationis) et la reconstruction d'une syntaxe latine au XVI ${ }^{\mathrm{e}}$ siècle ». Langages, 92, 51-64.

COLOMBO TIMELLI, Maria (1995). « Un Ars minor français publié à la fin du XV siècle (Paris, Felix Baligault, s.d.). Introduction et édition ». Archives et Documents de la Société d'Histoire et d'Épistémologie des Sciences du Langage, Seconde série, t. 12, 95-111.

COLOMBO TIMELLI, Maria (1996). Traductions françaises de l'Ars minor de Donat au Moyen Âge (XIII $X V^{e}$ siècles). Florence, La Nuova Italia, 225-230.

COLOMBO TIMELLI, Maria (1998). «Dialogues et phraséologie dans quelques dictionnaires plurilingues du XVI ${ }^{\mathrm{e}}$ siècle ». Documents pour l'Histoire du Français Langue Étrangère et/ou Seconde, 22, 27-63.

GÓMEZ GÓMEZ, Jesús (2000). El diálogo renacentista. Madrid : Laberinto.

LILLO, Jacqueline (1998). «Évolution des dialogues dans les manuels de français publiés en Italie du XVII ${ }^{\mathrm{e}}$ siècle à la Seconde Guerre mondiale ». Documents pour l'Histoire du Français Langue Étrangère et/ou Seconde, 22, 99-124. 
MEYER, Paul (1870). « La manière de langage qui enseigne à parler et à écrire le français ». Revue critique d'histoire et de littérature, 29, 373-381.

MINERVA, Nadia (1998). « Les dialogues dans les manuels italiens. Enquête quantitative » (1625-1860). Documents pour l'Histoire du Français Langue Étrangère et/ou Seconde, 22, 83-98.

PELLANDRA, Carla (1998). « Le dialogue théâtral et la classe de langue ». Documents pour l'Histoire du Français Langue Étrangère et/ou Seconde, 22, 143-156.

PLUCHE, Abbé (1751). La mécanique des langues. Paris : Chez la veuve Estienne \& Fils.

STATI, Sorin (1998). « L'enseignement du dialogue ». Documents pour l'Histoire du Français Langue Étrangère et/ou Seconde, 32, 7-16.

\section{Annexe}

ASSIER, Alexandre (1862). Nouveau manuel du premier âge, rédigé par demandes et par réponses, et mis à la portée des commençantes. Paris : Guérin-Muller. $1873^{2}$.

AUPHIGNY-BEAUVAIS, D. (1809). Nouvelles Leçons de grammaire française et d'orthographe, par demandes et réponses. Chaulnes : L'auteur.

BERTHELOT, R. (1845). La Grammaire des écoles élémentaires, par demandes et réponses, avec sujets d'analyse et d'exercices. Au Mans : Monnoyer.

BEDOUIN, P.-L.-E. (1811). Sommaire de la grammaire française, par demandes et par réponses.. Avignon : L. Aubanet.

BOICHARD, F.-X. (1836). Abrégé de grammaire française, par demandes et réponses. Besançon : L'auteur.

BONNAIRE, A. (1829). Grammaire française des commençans, par demandes et par réponses, avec des tableaux synoptiques pour les verbes. Paris : Lecointe. Autres éditions : Paris : L. Hachette, $1832^{2}$ et $1834^{3}$.

BURIDANT, J.-B.-R. (1874). Nouvelle Grammaire française par demandes et par réponses dont chaque règle est rendue compréhensible par des exercices analysés, décomposés et raisonnés. Paris : Ch. Delagrave.

CÉNAT DE L'HERM, Théodore (1843). Précis élémentaire de grammaire française par demandes et par réponses. Paris : Société de Saint-Nicolas.

CHÉANT, Maurice (1839). La plus facile des petites grammaires françaises, par demandes et par réponses. Laval : Godbert.

COFFIGNY, C. (1828). Élémens de la grammaire française, par demandes et par réponses.

Commerci : impr. de Denis.

COTON, Louis (1852). La Grammaire simplifiée par demandes et par réponses. Lyon : Périsse, $1853^{2}$.

DELAVENNE, Henri $\left(1893^{6}\right)$. La Grammaire des jeunes enfants par demandes et par réponses. Paris : A. Taffin-Lefort.

DUHAUT, Joseph Manuel (1851). Manuel des écoles primaires, renfermant la grammaire française par demandes et par réponses, un traité d'analyse grammaticale, le dictionnaire des homonymes, l'abrégé de la géographie. Nancy : l'auteur.

DUPUY, A. (1871). La Petite grammaire française des petits enfants par demandes et par réponses. Carpentras : impr. de L. Devillario. 
DURAND, F.-L. $\left(1845^{3}\right)$. Petite grammaire musicale, ou Principes élémentaires de la musique, exposés par demandes et réponses. Paris : J. Meissonnier.

GLAND, P.-L. (1832). Questions sur la grammaire française par demandes et par réponses. Sedan : impr. de Suhaux.

GROSE, D. (1842). Abrégé méthodique de la grammaire française, par demandes et par réponses. Bar-le-Duc : A. Laguerre.

IRIART, A. (1840). Introduction à la langue française et à la langue basque, Grammaire française, par demandes et par réponses. Bayonne : impr. de Vve Cluzeau.

LANNEAU, Pierre-Antoine-Victor Marey de (1824). Grammaire élémentaire par demandes et par réponses. Paris : Institution de Sainte-Barbe. $1825^{2}$.

LARIVE et FLEURY (1925). L'Année préparatoire de grammaire, par demandes et par réponses, avec 223 exercices faciles. Paris : A. Colin.

LE TELLIER, Charles-Constant $\left(1822^{7}\right)$. Grammaire des commençants... par demandes et par réponses, par Charles-Constant Le Tellier. Paris : C. Le Tellier fils. Paris : Belin-Le Prieur et Morizot, $1841^{21} ; 1842^{22} ; 1844^{23} ; 1846^{24}$.

LE SAINT, V. (Abbé) (1817). Grammaire latine de Lhomond, à l'usage des collèges, revue... et mise par demandes et réponses. Saint-Brieuc : Prud'homme.

LHOMOND, Charles François (1812). Grammaire française, par demandes et réponses, rédigée selon les principes. Vitry-sur-Marne.

LHOMOND, Charles François $\left(1853^{23}\right)$. Grammaire française élémentaire... selon le texte de Lhomond... par demandes et par réponses, suivie d'un Dictionnaire de locutions vicieuses, d'Exercices sur les homonymes, d'un Cours de thèmes ou d'exercices orthographiques... Mirecourt : Humbert.

L. G. D. (1851). Grammaire française des commençants par demandes et par réponses. Nouvelle édition, par réponses. Nouvelle édition. Reims : Naudin.

L'HOTE $\left(1868^{46}\right)$. Grammaire française élémentaire... selon le texte de Lhomond... par demandes et par réponses. Corrigé des exercices, par M. Salomon. Mirecourt : Humbert.

LÓPEZ DE MAIRAN, Juan Tomás de (1799). Compendio nuevo de gramática francesa. Madrid : Josef Herrera.

MAGNIEZ de WOIMONT, Louis-François (1721). Extrait du Postulant, ou Explication des principaux termes de la grammaire, par demandes et par réponses, pour faciliter l'intelligence de la langue latine. Paris : C. Huguier.

MAUGER, Claude \& FESTEAU, Paul (1693). Nouvelle double grammaire françoise-angloise et angloise-françoise. Bruxelles : Eugenius Henricus Frix.

MAUREL, J. (1828). Élémens de la grammaire latine de Lhomond. Nouvelle édition, par demandes et par réponses... augmentée d'un Extrait des Mœurs et coutumes des Romains. Avignon : impr. de L. Aubanel.

MEURIER, Gabriel (1558). Conjugaisons, règles et instruction mout propres et necessairement requises, pour ceux qui désirent apprendre François, Italien, Espagnol et Flamen : dont la plupart est mise en manière d'Interrogation et Response. Anvers : Ian van Waesberghe. 
MOURIER, J.-Fr. \& M. ALLIGIER (1812). Grammaire française, élémentaire et raisonnée, par demandes et par réponses... avec des notions de logique et de rhétorique qui ont rapport à la grammaire. Paris : Lenormant.

NAUDIN, P. (fils) $\left(1853^{3}\right)$. Grammaire française par demandes et par réponses. Reims : A. Naudin. OGER, Jean Valentin (1801). Principes Élémentaires par Demandes et par Réponses. Paris : Lefort. PANET, L. $\left(1822^{2}\right)$. Grammaire française, par demandes et réponses, rédigée selon les principes de M. Lhomond... Vitry-le-François : Farochon.

PELETIER DU MANS, Jacques (1555). Dialogue de l'orthografe e prononciation françoese departi an deus livres. Lyon : Jan de Tournes.

POTIN, Y.-M. (1869). Grammaire française par demandes et par réponses, suivie d'un abrégé du système métrique à l'usage des écoles primaires, des classes primaires dans les lycées, les collèges. Morlaix : J. Haslé.

RAMUS, Pierre de (1572). Grammaire. Paris : Imprimerie d'André Wechel.

RESTAUT, Pierre (1730). Principes généraux et raisonnés de la grammaire française par demandes et par réponses. Paris : Lottin. $1750^{5}, 1756,1817 \ldots$

RESTAUT, Pierre (1732). Abrégé des Principes. Paris : J. Desaint.

REYMOND, S. (1811). Leçons de grammaire française, par demandes et réponses... avec des remarques et des notes, suivies d'un abrégé de syntaxe, d'analyse, de fautes contre la langue, et des principaux mots homonymes. Lyon : Yvernault et Cabin.

RIBOURT, F. (1824). Grammaire latine simplifiée et mise dans un nouvel ordre, par demandes et réponses, avec un traité de la préposition, une méthode d'analyse et construction des phrases et la marche à suivre pour traduire du latin en français. Paris : A. Delalain.

ROULLEAUX, Mme (1833). Grammaire française à l'usage des pensionnats de demoiselles. Rennes : impr. de J.-M. Vatar.

SALAZAR, Ambrosio de (1614). Expexo general de la gramática en diálogos [...]. Rouen : Adrien Morront.

SENGLER, Antoine (S.J., Le P.) (1880). Petite grammaire des enfants, par demandes et par réponses. Lille : impr. de J. Lefort.

$\mathrm{SCHM}$. $\left(\mathrm{an} \mathrm{XII}{ }^{2}\right)$. Abrégé de grammaire française, rédigé en demandes et réponses d'après les principes des meilleurs grammairiens. Metz : impr. de G. Lamort.

SOTOMAYOR, Baltasar de (1565). Grammatica con reglas muy prouechosas y necessarias para aprender a leer y escriuir la lengua Francesa conferida con la Castellana, con vn vocabulario copioso de las mesmas lenguas ... Alcala de Henares : en casa de Pedro de Robles y Francisco de Cormellas.

SOTOMAYOR, Juan de (1706). Llave capital con la qual se abre el curioso y rico thesoro de la lengua castellana. Leipzig.

STÉVENIN, A. (1833) $2^{\mathrm{e}}$ éd. Grammaire française théorique et pratique, par demandes et par réponses. Paris : impr. de P. Dupont et Laguionie. Mézières : impr. de Garet, 1832.

VALDÉS, Juan de (1984 [1533]). Diálogo de la lengua. Ed. Antonio Quilis Morales. Barcelona : Plaza y Janés. 
WAILLY, François de (an VI). Abrégé de la grammaire, rédigé en demandes et en réponses par un professeur au ci-devant collège de Metz. Metz : impr. de C. Lamort.

\section{Ouvrages anonymes}

(1859). Grammaire Française des commençants, par demandes et par réponses. Nouvelle édition. Reims : Mérieux.

(1863). Petit Extrait de grammaire française par demandes et par réponses. Toulouse : imprimerie de Troyes.

(1831). Nouvelle Grammaire française en forme de demandes et de réponses, à l'usage des écoles, par un instituteur primaire. Montmédy : Cuny.

(1843). Abrégé des grammaires françaises, par demandes et par réponses, par E. F. Auxerre : Imp. de Ch. Gallit.

\section{NOTES}

1. Pour une connaissance approfondie de ce genre textuel, nous renvoyons à l'article de J.-Cl. Chevalier (1998), qui décrit les caractéristiques et l'évolution de ce type discursif dans ses usages didactiques.

\section{RÉSUMÉS}

Dans cet article, nous posons que les grammaires par demandes et réponses s'inscrivent dans un type d'ouvrages où les auteurs se servent d'une présentation dialoguée comme une forme discursive ou littéraire adaptée à la finalité qu'ils poursuivent. Après avoir répertorié ce type de grammaires (XVIe-XIXe siècles), nous analysons les particularités de ce mode de description/ présentation du contenu grammatical, en rapport aux modalités différentes de leur mise en discours, et nous postulons, en nous arrêtant de façon particulière sur le Diálogo de la lengua, de Valdés (1533) et les Principes de Restaut (1730), que ce type d'ouvrages s'insère dans l'histoire de l'enseignement $d u$ français comme une tentative de réunion de l'approche par règles et de l'approche par l'usage.

In this article we propose that the grammars written following a model of questions and answers are included in a type of works, which were practised since the antiquity, where the authors adopt a dialogue presentation as a discursive or literary way adapted to the ends they pursue. After listing this type of grammars (XVIth-XIXth centuries), we analyse the peculiarities of this methodology of description/presentation of the grammatical contents, establishing a relationship with the different modalities. We focus on Valdés' Dialogo de la lengua (1530) et Restaut Principles (1730), and we postulate that this type of works fits into the history of the teaching of French language as an attempt of meeting the approach through rules and by the use. 
INDEX

Keywords : colloquium, dialogue, grammars by demands and answers, teaching French, Pierre Restaut

Mots-clés : colloque, dialogue, enseignement du français, grammaires par demandes et réponses, Principes généraux et raisonnés de la grammaire française, Pierre Restaut

\section{AUTEUR}

\section{MARIA EUGENIA FERNÁNDEZ FRAILE}

Université de Granada 